\title{
High-throughput analysis of gene expression on tissue sections by in situ hybridization
}

\author{
Gregor Eichele ${ }^{\mathrm{a}, *}$, Graciana Diez-Roux ${ }^{\mathrm{b}}$ \\ ${ }^{a}$ Genes and Behavior Department, Max Planck Institute for Biophysical Chemistry, Am Fassberg 11, 37077 Goettingen, Germany \\ ${ }^{\mathrm{b}}$ Telethon Institute of Genetics and Medicine, Via Pietro Castellino 111, Naples 80131, Italy
}

\section{A R T I C L E I N F O}

Article history:

Available online 23 December 2010

\section{Keywords:}

Functional genomics

Gene expression analysis

\begin{abstract}
A B S T R A C T
Genome-scale sequencing projects, high-throughput RNAi screens, systematic gene targeting, and system-biology-based network predictions all depend on a validation of biological significance in order to understand the relevance of a particular finding. Such validation, for the most part, rests on low-throughput technologies. This article provides protocols that, in combination with suitable instrumentation, make possible a semi-automated analysis of gene expression on tissue sections by means of in situ hybridization. Knowledge of gene expression localization has the potential to aid, and thereby accelerate, the validation of gene functions.
\end{abstract}

(c) 2011 Elsevier Inc. All rights reserved.

\section{Description of theoretical basis and framework for the technique}

Elucidating the cellular localization of nucleic acids by means of in situ hybridization (ISH) dates back to the sixties (reviewed in [1]) and ever since this technique has been widely used to detect the location of specific nucleic acid sequences in their natural environment. ISH of the type discussed here involves the hybridization of in vitro synthesized RNA or DNA probes either to mRNA or microRNA present in tissue sections. Following hybridization, the location of the nucleic acid hybrid is visualized either by autoradiography or by colorimetry. Historically, ISH was carried out with radiolabeled probes, but the availability of hapten-tagged nucleotides and the development of sensitivity-increasing serial amplification procedures (see Section 1.2) have made non-radioactive ISH (NRISH) very popular. NR-ISH has several virtues, such as probe stability, avoidance of radioactive waste and access to a wide spectrum of color labels for simultaneous detection of more than one target. Although quantification of NR-ISH signal has also been performed [2], studies requiring highly accurate quantification of gene expression levels frequently draw on radioisotope-based autoradiography (e.g. [3]).

\subsection{High-throughputizing NR-ISH}

We briefly describe the measures implemented to standardize and increase the speed of NR-ISH to make it fit for high-throughput,

\footnotetext{
* Corresponding author.

E-mail addresses: geichel@gwdg.de (G. Eichele), diezroux@tigem.it (G. DiezRoux).
}

genome-scale work. Probes were designed and synthesized from freely available cDNA clone resources. For genes not represented in a public collection, templates were produced by PCR from cDNA using gene-specific primers. Signal detection was achieved using colorimetric detection of digoxigenin-tagged riboprobes of $\sim 1000 \mathrm{nt}$ in length. Gene expression levels of genes varied by several orders of magnitude. To enhance visualizion of transcripts with lower expression levels, a dual signal amplification procedure was implemented (see Section 1.2). To capture in full the complexity of expression patterns across an entire specimen while reducing time and efforts, multiple parallel sections were processed. E14.5 embryos, for example, are represented by a set of 24 sections, $200 \mu \mathrm{m}$ apart and each $25 \mu \mathrm{m}$ thick. To enable comparison between gene expression patterns, specimens were embedded in consistent orientation using custom-made freezing chambers. A scanning microscope was used to speed up imaging of expression patterns. Moreover, sections were placed at predefined positions that delineated a region of interest (ROI) imaged by the microscope. The ROI was defined by inserting standard-size microscope slides into a metal mask with characteristic rectangular windows delineating the ROI. Post-sectioning fixation, acetylation, paraffin removal, dehydration etc. were performed in a commercially available autostainer. Most importantly, ISH analyses were carried out by robotic equipment (Tecan GenePaint ${ }^{\mathrm{TM}}$ ). These robots consist of a Freedom $\mathrm{Evo}^{\mathrm{TM}}$ liquid handling platform equipped with up to four temperature-controlled racks that accommodate flow-through hybridization chambers. Reactants and washing solutions were applied to the chamber using microprocessor-controlled pipettes of the liquid handling platform. Such automation of NR-ISH has several advantages. The process can be carried out with little human intervention, the reagent addition procedures are standardized, temperature is 
controlled, and multiple repeats of reaction and washing steps can be implemented. Depending on section size, $\sim 1000$ tissue sections can be analyzed in a $24 \mathrm{~h}$ turnaround using robotic NR-ISH.

\subsection{Detection method}

In nearly all large-scale ISH projects ([4]; an exception is the Brain Gene Expression Map, BGEM, [5]), expression patterns were determined with digoxigenin-tagged riboprobes. The NR-ISH described here is based on dual signal amplification (reviewed in $[6,7])$ and approaches the sensitivity of radioisotopic ISH as it can detect as few as three transcripts per cell [8]. In the dual amplification strategy, termed "catalyzed reporter deposition" (CARD), hapten-tagged RNA (or DNA) probe is hybridized and subsequently detected in situ with an antihapten antibody conjugated to horseradish peroxidase (e.g., POD). The catalytic activity of the POD is used to activate a tyramide-biotin (TB) complex so that for each bound antihapten antibody, multiple biotinylated tyramine molecules covalently attach to reactive groups in the vicinity of the probe Thereafter, biotin is detected with an avidin-alkaline phosphatase conjugate, whose enzymatic activity converts nitro blue tetrazolium (NBT) and 5-bromo-4-chloro-3-indolyl-phosphate (BCIP) substrates into an insoluble, stable, blue-purple indigo and formazan dye precipitate. A second round of TB amplification can be carried out prior to initiation of the color reaction in order to strengthen the signal. Colorimetric detection using tyramide-fluoresceinisothiocyanate (FITC) instead of tyramide-biotin is indicated if tissues contain endogenous biotin. Alternatively, endogenous biotin can be blocked using a biotin blocking kit (Vector Laboratories).

\section{Description of methods}

The diagram in Fig. 1 outlines the sequential procedures that constitute the high-throughput NR-ISH process. Each individual step is described in detail below.

\subsection{Materials}

\subsubsection{Hardware}

2.1.1.1. Freezing chamber. (Fig. 2A) A custom-made specimen freezing chamber consists of transparent plexiglas side walls that are placed into grooves milled into a copper base plate. To fasten the sidewalls from the top, each sidewall is equipped with two stainless steel pegs that fit into holes located in an aluminum plate capping the chamber. This plate is fastened to the vertical stainlesssteel rods with two clips.

\subsubsection{Microtome. Leica CM3050S cryostat.}

2.1.1.3. Slides and slide holder. (Fig. 2B) A SuperFrost ${ }^{\circledR}$ Plus slide is placed into a custom-made holder characterized by predefined "windows" (regions of interest, ROIs) for accurate placement of tissue sections. A polyoxymethylene spring holds the slide in place.

2.1.1.4. Autostainer. Leica autostainer XL (or equivalent product from another manufacturer) equipped with metal (or plastic) slide racks and solution containers.

2.1.1.5. Genepaint ${ }^{\mathrm{TM}}$ hardware. (Fig. $2 \mathrm{C}$ and D) The Genepaint instrument needed for automated ISH consists of (1) a Tecan Freedom $\mathrm{Evo}^{\mathrm{TM}}$ solvent delivery robot, (2) flow-through hybridization chambers, (3) up to four temperature-controlled chamber racks, each accommodating 48 hybridization chambers, (4) a bath to pre-warm the stringency wash solutions, (5) circulating water

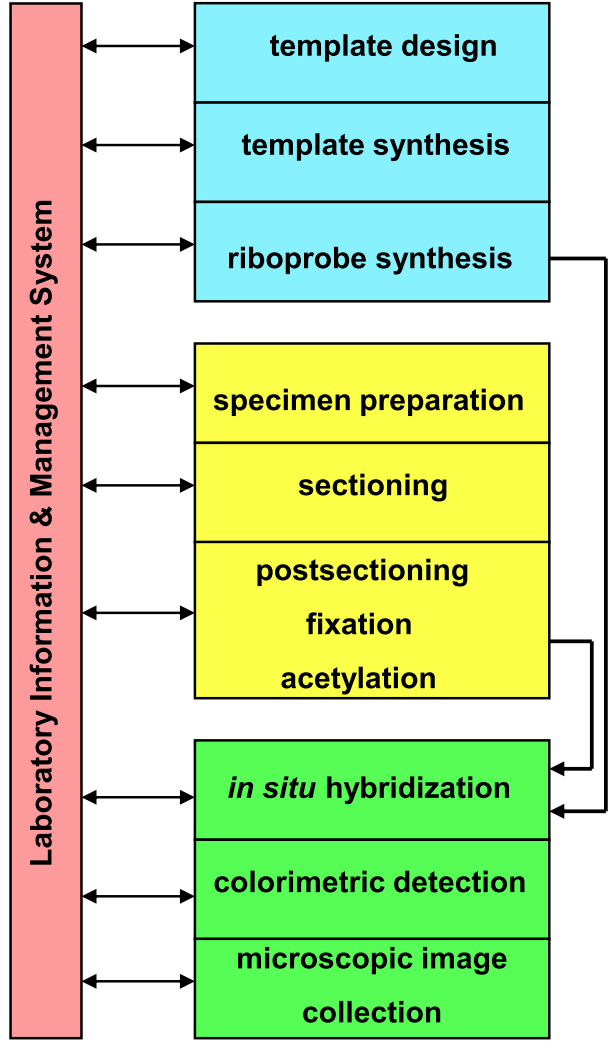

Fig. 1. Organization of the production pipeline for NR-ISH. High-throughput in situ hybridization with non-radioactive probes consists of three modules, each performed by a different team. Module 1 (bioinformatics/molecular biology) engages in probe design and synthesis (pale blue rectangles). Module 2 (histology) personnel collects and sections tissues (yellow rectangles). Module 3 tasks are in situ hybridization and image data collection (green rectangles). It is critical that the three modules are interconnected by a laboratory information and process management system that supervises and coordinates the flow of materials and data through the production pipeline.

baths that maintain a user-defined temperature in the chamber racks and the stringency wash bath, and (6) a series of solution containers located on the robot's platform. The solvent delivery system is capable of controlling any number of external devices, including the water baths.

\subsubsection{Coverslipper. Used for automated application of coverslips.}

\subsubsection{Reagents}

The list below contains only non-standard reagents. In all other cases molecular biology grade reagents are used.

(1) Coverslipping medium Hydro-Matrix (Micro-Tech-Lab, Graz, Austria; (http://www.lmscope.com/produkt22/Einschlussmittel_en.shtml).

(2) Hybridization buffer (Applied Biosystems, B8807G).

(3) Blocking reagent (Perkin Elmer, FP1012).

(4) Blocking reagent for nucleic acid hybridization and detection (Roche Applied Science, 11096176001).

(5) Anti-digoxigenin-POD, Fab fragments from sheep (Roche Applied Science, 11207733910).

(6) NeutrAvidin Protein, alkaline phosphatase conjugated (Thermo Scientific, 31002).

(7) TSA ${ }^{\mathrm{TM}}$ Biotin Tyramide Reagent Pack Reagents for 10003000 slides (Perkin Elmer, SAT700B001EA).

(8) 5-Bromo-4-chloro-3-indolyl-phosphate (Roche Applied Science, 11383221001). 

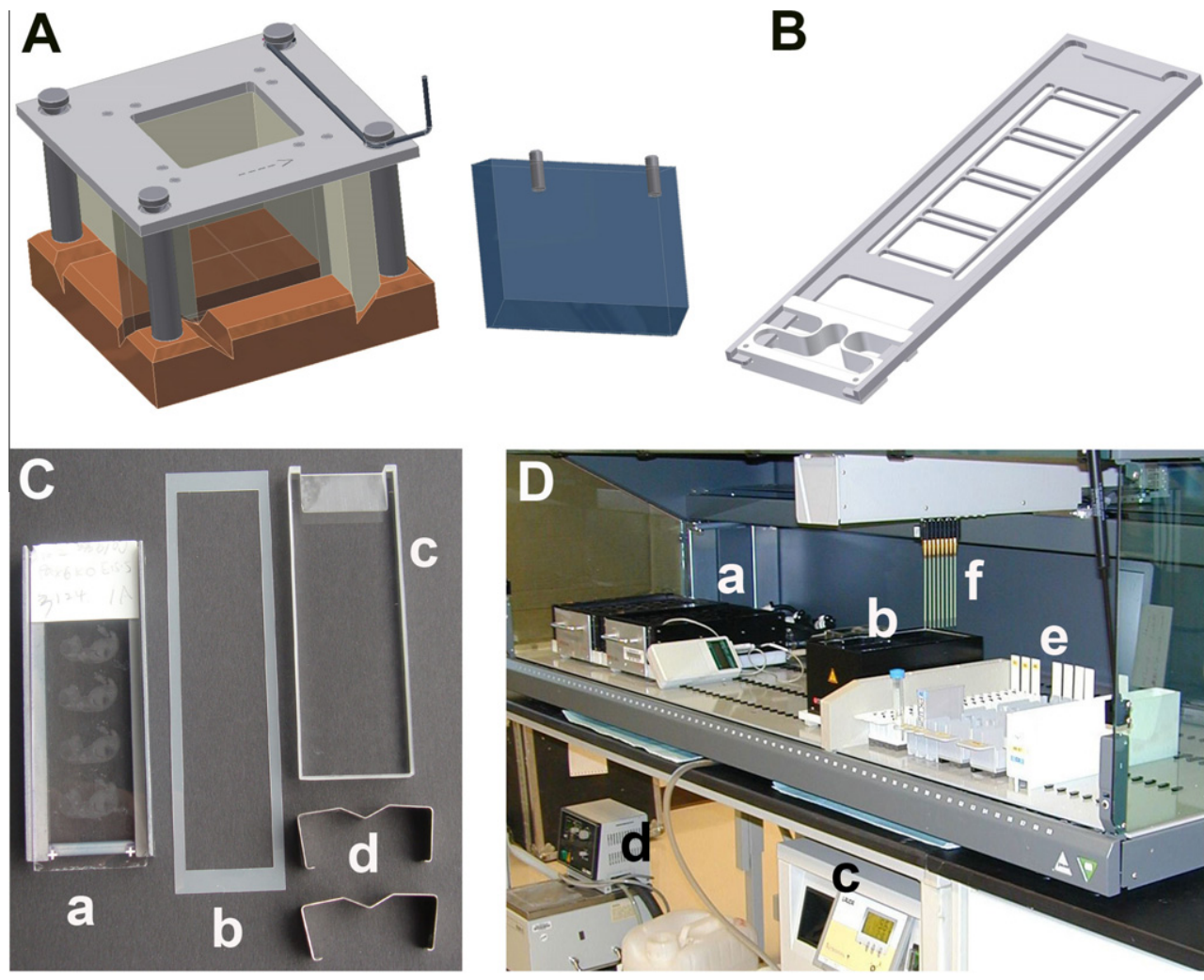

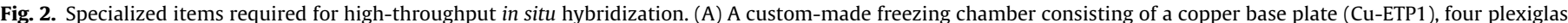

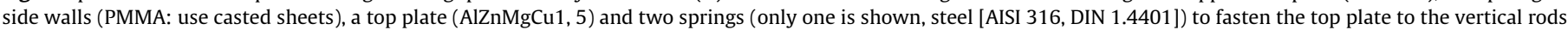

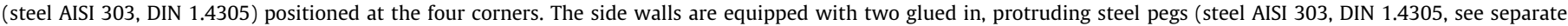

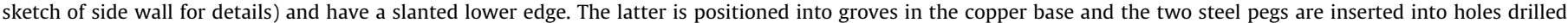

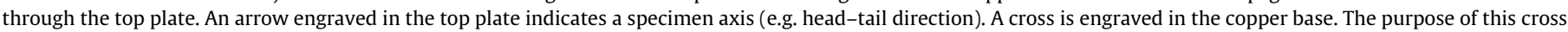

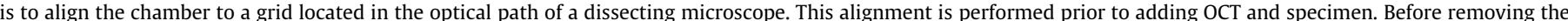

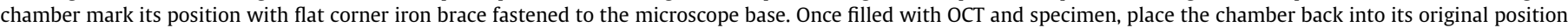

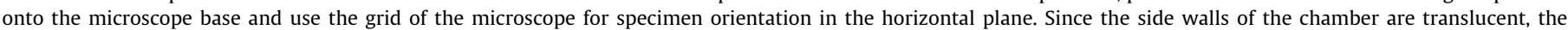

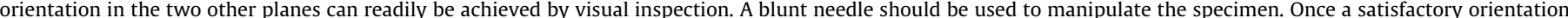

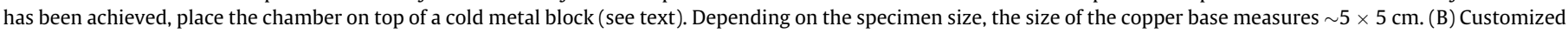

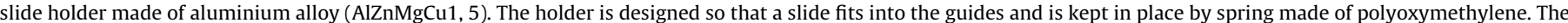

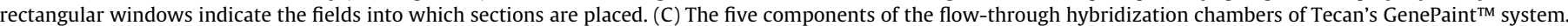

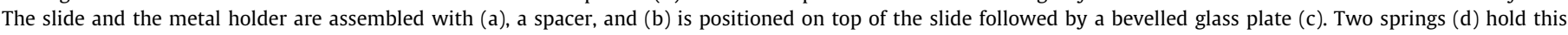

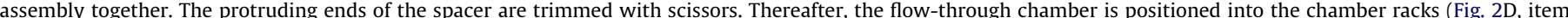

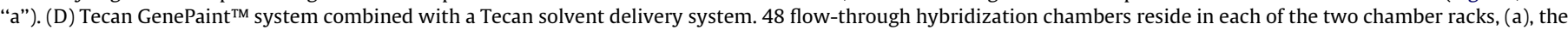

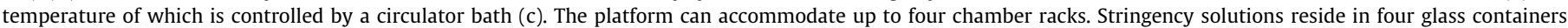

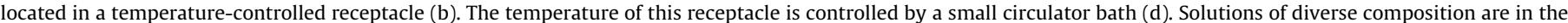
solution container area (e) and are added by solution delivery pipettes (f).

(9) 4-Nitro blue tetrazolium (Roche Applied Science, 11383213001).

(10) Streptavidin/biotin blocking kit (Vector Laboratories SP2002).

(11) TSA ${ }^{\text {TM }}$ Plus Fluorescein System for 250-750 slides (Perkin Elmer, NEL741B001KT).

(12) Anti-Fluorescein-POD, Fab fragments from sheep (Roche Applied Science, 11426346910).

(13) Levamisole hydrochloride (Sigma-Aldrich, L9756).

(14) Proteinase K, recombinant, PCR Grade Solution (Roche Applied Science, 03115887001).

\subsection{Procedures}

\subsubsection{Template design and riboprobe synthesis}

Tackling large-scale expression analyses requires suitable DNA templates and riboprobes. Three main criteria need to be met by ISH probes: (1) Probes should not cross-hybridize with other transcribed sequences. Due to the significant similarity sometimes found within the coding sequence of paralogous genes, the 3'UTR of transcripts is the region of choice for template design. In order to assess for specificity, template sequences were compared to the organism-specific gene reference databases, available at ENSEMBL (http://www.ensembl.org/index.html), Genome Browser at the University of California, Santa Cruz (genome.ucsc.edu) and Entrez Gene databases (http://www.ncbi.nlm.nih.gov/) using BLAST (http://www.ncbi.nlm.nih.gov/BLAST/), or BLAT analyses. (2) In a survey study, the ISH template should cover most of the (known) splice variants, unless a particular isoform is investigated. (3) The template length should range between 600 and 1000 nucleotides. Because the above criteria cannot be always simultaneously satisfied, specificity should take priority over probe size. Semi-automated routines enable identification of an appropriate probe for the majority of the $>20,000$ mouse genes. Valuable sources of templates are the publicly available cDNA clone libraries, such as the IMAGE collection and the Mammalian Gene Collection (MGC) (http://mgc.nci.nih.gov/). These cDNA clones are used in genome-scale projects to generate ISH templates, preferentially by PCR. After sequence verification, the cDNA inserts of these clones serve as direct templates for PCR using universal promoter 
sequence-specific (T3, T7, SP6) oligonucleotide primers. Alternatively, templates are generated from cDNA clones using a combination of vector-specific and transcript-specific oligonucleotide primers. If cDNA clones are not available, templates are generated by isolating mRNA from appropriate tissues followed by reverse transcription to generate cDNA. The latter is PCR-amplified with transcript-specific oligonucleotide primers cDNA (for optimal primer selection see http://frodo.wi.mit.edu/primer3/). Alternatively, PCR on genomic DNA with gene-specific primers can be carried out. The latter option may be preferable if a designed template corresponds to a DNA fragment colinear with genomic DNA.

2.2.1.1. Amplification from $c D N A$. Templates are generated from a cDNA cocktail derived by reverse transcription of mRNA from E10.5 and E14.5 mouse embryos and P7 and P56 mouse brains. The PCR uses specific forward and reverse primers extended at their 5' end with either T7 or SP6 promoter sequences (T7, GCGTAATACGACTCACTATAGGG; SP6, GCGATTTAGGTGACACTATAG; the three underlined extra bases facilitate RNA polymerase binding). For example, the Ear2 gene specific primers are as follows: Ear2 forward, T7-5'-ACCGAGTATGTGCGTGCCCAG-3'; Ear2 reverse, SP6-5'-TGGTGCCCTCCGTGGACCATG-3'. Temperature gradient PCR $\left(55-65^{\circ} \mathrm{C}\right)$ is carried out with six cDNA aliquots, containing $2 \mu \mathrm{l}$ of forward and reverse primers $(5 \mathrm{pmol} / \mu \mathrm{l}$, e.g., MWG Biotech), $2 \mu \mathrm{l}$ of cDNA, $2 \mu \mathrm{l}$ of dNTPs ( $2 \mathrm{mM}$, Roche Applied Science), $10 \times$ PCR buffer (Qiagen), $5 \times \mathrm{Q}$ enhancer (Qiagen), and $0.1 \mu \mathrm{l}$ Taq polymerase ( $\mathrm{U} / \mu \mathrm{l}$, Qiagen), and water to a volume of $10 \mu \mathrm{l}$. PCR conditions are as follows:

2 Min initial template denaturation at $94^{\circ} \mathrm{C}, 35$ cycles with $20 \mathrm{~s}$ denaturation $\left(94^{\circ} \mathrm{C}\right), 20 \mathrm{~s}$ primer annealing $\left(55-65^{\circ} \mathrm{C}\right), 1 \mathrm{~min}$ per kilobase of elongation $\left(72^{\circ} \mathrm{C}\right)$, and a final elongation cycle for $9 \mathrm{~min}$ at $72{ }^{\circ} \mathrm{C}$. The resulting product is analyzed and purified on an agarose gel, and the desired band is extracted using the Qiagen gel extraction kit. Initial PCR products are reamplified in a reaction consisting of $10 \mu \mathrm{l}$ each of the gene-specific forward and reverse primer $(5 \mathrm{pmol} / \mu \mathrm{l}), 20-50 \mathrm{ng}$ of PCR product, $10 \mu \mathrm{l}$ of dNTPs $(2 \mathrm{mM}), 10 \times$ PCR buffer, $5 \times \mathrm{Q}$ enhancer, and $0.5 \mu \mathrm{l}$ Taq polymerase, and water to a total volume of $100 \mu \mathrm{l}$. PCR conditions are described above, except that the primer annealing temperature is that determined to be optimal in the gradient PCR. Templates are purified using QIAquick PCR purification spin columns (Qiagen) and aliquots ( $\sim 200 \mathrm{ng}$ ) of the purified DNA (usually $\sim 10 \mu \mathrm{g}$ ) are analyzed on an agarose gel. PCR products are sequence-verified before in vitro transcription.

2.2.1.2. Amplification from $c D N A$ clones. PCR reactions are performed in a $100 \mu \mathrm{l}$ total volume with final concentrations of $1 \times$ Taq buffer, $1.5 \mathrm{M}$ Betaine, $0.2 \mathrm{mM}$ dNTPs, $5 \mathrm{U}$ Taq polymerase, $10 \mathrm{U}$ of Pfu DNA polymerase and $0.5 \mu \mathrm{M}$ of each primer. It is recommended to perform a sequence verification.

2.2.1.3. RNA probe synthesis. In vitro transcription is carried out in a cocktail of $2 \mu \mathrm{l}$ of rATP, rCTP, rGTP (10 mM, Roche Applied Science), $1.3 \mu \mathrm{l}$ of rUTP $(10 \mathrm{mM}), 0.7 \mu \mathrm{l}$ of digoxigenin-UTP $(10 \mathrm{mM}$, Roche Applied Science), $2 \mu \mathrm{l}$ of dithiothreito $(0.75 \mathrm{M}), 1 \mu \mathrm{l}$ of ribonuclease inhibitor (40 U/ $\mu \mathrm{l}$ MBI Fermentas), $1 \mu \mathrm{g}$ of DNA template, $0.5 \mu \mathrm{l}$ of RNA polymerase (T7: $50 \mathrm{U} / \mu \mathrm{l}, \mathrm{SP} 6: 20 \mathrm{U} / \mu \mathrm{l}$, both New England Biolabs), $5 \times$ transcription buffer and DEPC water to a final volume of $20 \mu$ l. The reaction mix is incubated at $37^{\circ} \mathrm{C}$ for at least $150 \mathrm{~min}$ followed by a 20 -min incubation with a DNase $\mathrm{I} / \mathrm{MgCl}_{2}$ mix to remove the DNA template $(1.6 \mu \mathrm{l}$ of $300 \mathrm{mM} \mathrm{MgCl} 2,2 \mu \mathrm{l}$ of DNase I [10 U/ $\mu$ l, Roche Applied Science], $16.4 \mu$ l of DEPC water). RNA is ethanol precipitated, re-dissolved in $25 \mu \mathrm{l}$ of DEPC water while vigorously shaking at room temperature. Two microliters of the RNA are analyzed on a $1 \%$ agarose gel, and $1 \mu \mathrm{l}$ is used to determine concentration in a NanoDrop instrument. Probes are diluted with hybridization mix to $100 \mathrm{ng} / \mu \mathrm{l}$ and stored at $-20^{\circ} \mathrm{C}$. Before use for ISH, riboprobes are diluted in hybridization buffer to a concentration of $150-400 \mathrm{ng} / \mathrm{ml}$.

\subsubsection{Tissue preparation and sectioning}

2.2.2.1. Fresh frozen section protocol. Tissues such as E14.5 embryos, and postnatal or adult brains, are collected in ice-cold phosphate buffered saline, blotted dry with a filter paper, immersed in icecold OCT 4583 (Tissue-Tek) for $\sim 5 \mathrm{~min}$, and transferred into an OCT-filled, freezing chamber (Fig. 2A). The embryo specimen is oriented and the chamber placed on top of a metal block immersed in a mixture of dry ice and ethanol $\left(-70^{\circ} \mathrm{C}\right)$ and left there until freezing OCT has reached the embryo. Thereafter, the chamber is transferred to a $-20^{\circ} \mathrm{C}$ freezer. A few hours later, the chamber is disassembled and the block is labeled with specimen number and a rostrally-pointing arrow and stored in a sealed plastic bag at $-80^{\circ} \mathrm{C}$. Before sectioning, the OCT block is equilibrated at $-20{ }^{\circ} \mathrm{C}$ for at least one day and frozen sections are cut in a cryostat with the chamber and object holder temperatures set so that the temperature in proximity of the specimen is approximately $-10^{\circ} \mathrm{C}$, measured with a thermometer placed near the specimen. Sections (20 or $25 \mu \mathrm{m}$ thick) are collected from eye-to-eye on SuperFrost ${ }^{\circledR}$ Plus slides that are clipped into slide holders (Fig. 2B). Once all four ROIs are occupied, slides are placed into metal slide racks. Slide racks are transferred into Zip-lock bags and stored overnight $\left(-20^{\circ} \mathrm{C}\right)$ in the presence of silica gel. Thereafter, the unthawed sections are fixed at room temperature in $4 \%$ paraformaldehyde for 15-30 min, washed two times for $2 \mathrm{~min}$ in $0.9 \%$ sodium chloride acetylated twice for 5 min each in fresh $0.25 \% \mathrm{v} /$ $\mathrm{v}$ acetic anhydride in $0.1 \mathrm{M}$ triethanolamine ( $\mathrm{pH} 8.0$ ), and dehydrated in the autostainer in an ethanol series ending with $100 \%$ ethanol. For long-term storage, air-dried slides are stored at $-80^{\circ} \mathrm{C}$ in sealed slide boxes containing desiccant.

2.2.2.2. Paraffin section protocol. Tissues (e.g., embryonic E17.5 or adult, P84 mouse kidneys) are collected in ice-cold phosphate buffered saline (PBS) and directly transferred into fixing solution. E17.5 kidneys are fixed for $3 \mathrm{~h}$ at $4{ }^{\circ} \mathrm{C}$ in a mix of $60 \mathrm{ml}$ absolute ethanol, $30 \mathrm{ml}$ formaldehyde (37\% stabilized in methanol), and $10 \mathrm{ml}$ glacial acetic acid. Adult kidneys are fixed for three days at $4{ }^{\circ} \mathrm{C}$ in $4 \%$ paraformaldehyde in PBS replaced daily. Specimens are then dehydrated in an alcohol series (35\%; 50\%; 75\%; two times $100 \%$ ethanol; two times in xylene) and embedded in paraffin (xylene/paraffin; two times in paraffin). Kidneys are kept in each step for 30-60 min (E17.5) or one day (P84). Exceptions are embryonic kidneys, which start the series in $75 \%$ ethanol and for easier visualization within the block they are stained with eosin in the first xylene step. Also, they are kept in paraffin overnight. Blocks are stored at room temperature. The first third of the long axis of each embedded kidney is trimmed and $8 \mu \mathrm{m}$ transverse sections are collected on SuperFrost ${ }^{\circledR}$ Plus slides. Sections are deparaffinized and rehydrated $\left(2 \times 10 \mathrm{~min}\right.$ X-TRA-Solv ${ }^{\circledR}$ [a xylene substitute]; $2 \times 10$ min $100 \%$ ethanol; 2 min 95\%, 80\%, 70\%, 50\%, 30\% ethanol each; PBS) using an autostainer. Thereafter, the sections are fixed at room temperature in 4\% paraformaldehyde for 15-30 min, acetylated twice for $5 \mathrm{~min}$ each in fresh $0.25 \% \mathrm{v} / \mathrm{v}$ acetic anhydride in $0.1 \mathrm{M}$ triethanolamine ( $\mathrm{pH} 8.0$ ), and dehydrated in an ethanol series ending with $100 \%$ ethanol. Air-dried sections are stored at $-80^{\circ} \mathrm{C}$ in sealed slide boxes containing desiccant.

\subsubsection{In situ hybridization}

2.2.3.1. Assembly of hybridization chambers and prehybridization. Carried out on the first day. Depending on the number of slides, the assembly of chambers should start around noon or later. 
1. Slides are thawed at $37^{\circ} \mathrm{C}$ for $30 \mathrm{~min}$ while they are still sealed inside slide boxes. Thereafter, they are assembled into flow-through hybridization chambers (Fig. 2C) and placed into the chamber racks located on the Tecan platform (Fig. 2D, item "a"). From here onward, all reagents are added using liquid handling system pipettes. The prehybridization process starts by incubating the slides five times for $5 \mathrm{~min}$ with methanol containing $0.6 \%$ hydrogen peroxide (peroxide is added to methanol just before use). Pipetted solution volumes are $300 \mu$ unless noted otherwise. The process further continues with the following steps:

2. Incubate seven times for 5 min with PBS.

3. Incubate twice for 5 min with $0.2 \mathrm{~N} \mathrm{HCl}$.

4. Incubate four times for $5 \mathrm{~min}$ with PBS.

5. Incubate once for $5 \mathrm{~min}$ with $400 \mu \mathrm{l}$ of proteinase $\mathrm{K}$ buffer (50 mM Tris, $5 \mathrm{mM}$ ethylenediaminetetraacetic acid [EDTA], pH 8.0).

6. Incubate twice for $10 \mathrm{~min}$ with $2 \mu \mathrm{g} / \mathrm{ml}$ proteinase $\mathrm{K}$ in proteinase $\mathrm{K}$ buffer. Proteinase $\mathrm{K}$ is freshly added to the buffer as step 5 commences, mixed, and placed onto the Tecan platform. IMPORTANT: Different developmental stages and tissues require adjustment to proteinase $\mathrm{K}$ concentration. For paraffin sections of adult kidney, for example, the concentration is $10 \mu \mathrm{g} / \mathrm{ml}$.

7. Incubate seven times for 5 min with PBS.

8. Incubate twice for $10 \mathrm{~min}$ with $4 \%$ paraformaldehyde (PFA).

9. Incubate seven times for 5 min with PBS.

10. Incubate with hybridization buffer. Hybridization buffer may be preheated at $65^{\circ} \mathrm{C}$ in an incubator oven for $\sim 10$ min. After cooling down to room temperature, add dithiothreitol (Sigma) to $1.5 \mathrm{mg} / \mathrm{ml}$.

11. A second aliquot of hybridization buffer is added, and incubation is continued at room temperature for 15 more min, after which the script increases the temperature of the chamber rack to $64^{\circ} \mathrm{C}$.

2.2.3.2. Hybridization step. Carried out during the first part of the night from day 1 to day 2 .

RNA probe (150-400 ng/ml final concentration) is dissolved in hybridization buffer prepared as described in step 10 (see above) and 250-300 $\mu \mathrm{l}$ are added to each hybridization chamber by the script. After $2.5 \mathrm{~h}$, a fresh aliquot of probe is pipetted by the script and the hybridization process is continued for $3 \mathrm{~h}$ at $64{ }^{\circ} \mathrm{C}$.

2.2.3.3. Stringency washes. Carried out at $62{ }^{\circ} \mathrm{C}$ during the second part of the night from day 1 to day 2 .

On day $1,5 \times$ and $0.1 \times$ SSC are degassed in the vacuum oven at room temperature. Formamide-containing solutions are degassed by heating them to $65^{\circ} \mathrm{C}$ in a water bath in a closed bottle (bottles should not be filled to the top) until the solutions are slightly turbid (phase separation from Tween); this process may take 1-2 h. These solutions are then left to cool to room temperature (RT).

Proper heating of stringency wash solutions on the robot platform in a dedicated temperature-controlled receptacle is critical (Fig. 2D, item b). The receptacle is warmed up by a circulator bath (item d) and depending on the capacity of this bath and the length of the tubing that delivers the water to the receptacle, the time it takes to reach $65^{\circ} \mathrm{C}$ may be $1-2 \mathrm{~h}$. This delay needs to be empirically determined when the system is set up and the robot's script need to ensure that the water bath initiates heating in a timely manner. The process further continues as follows:

1. Incubate five times for $5 \mathrm{~min}$ with $5 \times \operatorname{SSC}(0.15 \mathrm{M} \mathrm{NaCl}$, $0.015 \mathrm{M}$ sodium citrate, $\mathrm{pH} 7.0$ ).
2. Incubate five times for 10 min with $2 \times$ SSC containing $50 \%$ formamide.

3. Incubate five times for 12 min with $1 \times$ SSC containing $50 \%$ formamide.

4. Incubate four times for $8 \mathrm{~min}$ with $0.1 \times$ SSC. During the last cycle the script ramps-down the chamber rack temperature to $25^{\circ} \mathrm{C}$.

2.2.3.4. Immunohistochemical detection of riboprobe. Morning of day 2. Solutions up to the tyramide signal amplification step are placed on the robot platform and left standing there overnight. Solutions required after this step are placed onto the platform in the morning of day 2 . The process continues as follows:

1. Incubate four times for $5 \mathrm{~min}$ with $\mathrm{NTE}(500 \mathrm{mM} \mathrm{NaCl}$, $10 \mathrm{mM}$ Tris, 5 mM EDTA, pH 7.6).

2. Incubate six times for $5 \mathrm{~min}$ in $20 \mathrm{mM}$ iodoacetamide (Sigma) in NTE. This solution is made freshly on day 1 , degassed for $30 \mathrm{~min}$ in a vacuum oven at room temperature and placed onto the platform.

3. Incubate four times for 5 min with NTE.

4. Incubate two times at room temperature for 5 min each with TN (100 mM Tris, 150 mM NaCl, pH 7.6).

5. Incubate six times for $5 \mathrm{~min}$ in sheep serum in TN. On day 1 , mix 4\% (w/v) heat-inactivated sheep-serum (Sigma-Aldrich S2263, place bottle with serum into a $60{ }^{\circ} \mathrm{C}$ waterbath, equilibrate temperature and continue incubation for 30 more minutes, swirl bottle from time to time until $30 \mathrm{~min}$ have elapsed, let serum cool down, filter with a Whatman paper filter, aliquot serum) in TN, vacuum filter through a $0.45 \mu \mathrm{m}$ HV Durapore membrane (Millipore), degas in a vacuum chamber, and place on platform.

6. Incubate four times for 5 min with $\mathrm{TN}$.

7. Incubate two times at room temperature for $10 \mathrm{~min}$ with TNB blocking buffer (100 mM Tris, $150 \mathrm{mM} \mathrm{NaCl}, 0.5 \%$ (w/ v) blocking reagent (Perkin Elmer, FP1012)). TNB is prepared on day 1 as follows: An Erlenmeyer flask containing the TNB mixture is placed on a magnetic stirrer set to $60^{\circ} \mathrm{C}$. Stir for $1 \mathrm{~h}$ until substances are dissolved. Continue stirring for $1 \mathrm{~h}$ at room temperature, and then filter through a $0.45 \mu \mathrm{m} \mathrm{HV}$ Durapore membrane and degas.

8. Incubate two times for $5 \mathrm{~min}$ with TN solution.

9. Incubate two times for $5 \mathrm{~min}$ with maleate wash buffer (MWB; $100 \mathrm{mM}$ maleate, $150 \mathrm{mM} \mathrm{NaCl}, \mathrm{pH}$ 7.5). This solution is prepared on day 1 .

10. Incubate two times for 10 min with a day 1-prepared MWB containing $1 \%(\mathrm{w} / \mathrm{v})$ blocking reagent (BR, Roche Applied Science, 11096176001). To prepare this reagent, an Erlenmeyer flask containing $1 \% \mathrm{BR}$ mixture is placed on a magnetic stirrer set to $60^{\circ} \mathrm{C}$. Stir for $1 \mathrm{~h}$ until substances are dissolved. Continue stirring for $1 \mathrm{~h}$ at room temperature, and then filter through a $0.45 \mu \mathrm{m}$ HV Durapore membrane and degas.

11. Incubate two times for $5 \mathrm{~min}$ with MWB.

12. Incubate two times for $5 \mathrm{~min}$ with TN solution.

13. Incubate three times for 5 min with a day 1-prepared TMN solution (0.1 M Tris, 0.05 M MgCl2, 0.1 M NaCl, pH 9.5). Degas for $30 \mathrm{~min}$ in a vacuum oven at RT.

14. Incubate four times for $5 \mathrm{~min}$ with TN solution.

15. Incubate four times for $10 \mathrm{~min}$ each with TNB blocking buffer.

16. Incubate two times for 30 min with a day 1-prepared antidigoxigenin-POD (Roche Applied Science) diluted to a final concentration of $0.3 \mathrm{U} / \mathrm{ml}$ (this can be adjusted by $\pm 20 \%$, if background seems too high) in degassed TNB.

17. Incubate six times for 5 min with TN. 
18. Incubate for $20 \mathrm{~min}$ with $250-300 \mu$ tyramide-biotin diluted 1:50 with TSA amplification diluent. This solution is prepared on day 2 and prewarmed to RT and is used immediately.

19. Incubate six times for 5 min with MWB.

20. Incubate two times for $30 \mathrm{~min}$ with a day 2-prepared neutravidin-alkaline phosphatase (N-AP, Thermo Scientific) conjugate, diluted to a final concentration of $1.2 \mathrm{U} / \mathrm{ml}$ in $1 \% \mathrm{BR} / \mathrm{MWB}$ (preparation of $1 \% \mathrm{BR} / \mathrm{MWB}$ is described in point 10 above).

21. Incubate six times for 5 min with MWB.

22. Incubate four times for $5 \mathrm{~min}$ with $\mathrm{TN}$.

23. Incubate two times for $5 \mathrm{~min}$ with TMN containing levamisol $(0.5 \mathrm{mg} / \mathrm{ml}$, Sigma $)$.

24. Incubate three times for $10 \mathrm{~min}$ in chromogenic reagent that is prepared as follows. To TMN add solutions of 5-bromo-4chloro-3-indolyl- phosphate (final concentration is 0.15$0.2 \mathrm{mg} / \mathrm{ml}$ ), nitro blue tetrazolium (NBT; final concentration is $0.4 \mathrm{mg} / \mathrm{ml}$ ) and levamisol (final concentration is $0.5 \mathrm{mg} /$ $\mathrm{ml}$ ). The color reaction step can be extended depending on signal strength.

25. Wash four times with $400 \mu \mathrm{l}$ of system liquid water (provided by the robot).

26. Incubate for $5 \mathrm{~min}$ with NTE.

27. Incubate for $10 \mathrm{~min}$ with $200 \mu \mathrm{l}$ of $4 \%$ PFA.

28. Wash four times with $400 \mu \mathrm{l}$ of system liquid water.

29. Disassemble hybridization chambers while they are immersed in dust-free water, air-dry slides overnight, and coverslip them with aqueous Hydro-Matrix medium. Squeeze out bubbles and remove excess medium with a moist tissue paper.

\subsubsection{Analysis of micro RNAs}

The protocols used are similar to those described above for mRNA with the following exceptions:

(1) Locked nucleotide DNA probes haptenized with digoxygenin at either the 3' or 5' ends or both ends are used (e.g. Exiqon).

(2) Probe concentrations in the hybridization solution are 10$30 \mathrm{nM}$; the optimal concentration has to be determined for each probe.

(3) Hybridization and washing temperatures are $\mathrm{Tm}^{\text {calc }}$ $21 \pm 5^{\circ} \mathrm{C}$.
(4) The stringency wash solutions are composed as follows:

First wash: 5x SSC

Second wash: 10x SSC with $50 \%$ formamide

Third wash: $5 x$ SSC with $50 \%$ formamide

Fourth wash: $2 x$ SSC

\subsection{Quality issues}

The three factors primarily responsible for determining the quality of NR-ISH expression patterns are tissue quality, choice of probe, and strict adherence to an optimized protocol. While modifying the temperature for hybridization and/or the stringency wash can improve results to some extent, poor data are usually caused by inadequate tissue preparation and/or use of an unsuitable probe. For example, postnatal and, to an even greater extent, adult mouse brain can readily be prepared as fresh frozen cryo sections (in OCT) (see [4]). Likewise, mouse embryos from E12.5 onward can be prepared as fresh frozen material. By contrast, chicken embryonic brain from Hamburger-Hamilton stage 3036 requires immersion fixation in PFA, followed by cryoprotection in sucrose, followed by embedding and freezing in OCT.

Water-rich organs such as the adult kidney are not suitable as fresh-frozen preparations because of ice formation in the kidneys disrupts kidney tubules, thereby rupturing epithelial cells. The resulting poor histology causes a substantial loss in cellular resolution, which can be circumvented by using paraffin-embedded kidneys. With paraffin sections the concentration of proteinase $\mathrm{K}$ is about 5 times higher than that used for fresh frozen tissue. The exact enzyme concentration needs to be optimized and depends on tissue type and section thickness. Moreover, the various brands and lots of proteinase $\mathrm{K}$ may differ in their specific activity. When planning a scaling up of NR-ISH, it should be kept in mind that procedures requiring elaborate tissue preparation such as perfusion are usually too labor-intensive and are therefore unsuitable for high-throughput processing.

Unsatisfactory results may also arise from deviations from the standardized procedures described above. The most common problem is the presence of circular spots in which no signal is detected. This is caused by the formation of small air bubbles that attach to the section and stay in place despite the continuous addition of new solutions. Air bubble formation results from reduction of gas solubility as solution temperature increases. Thus, all solutions must be degassed. In addition, stringency wash solutions must be preheated just before use to $65^{\circ} \mathrm{C}$ in a bath (Fig. 2D, item
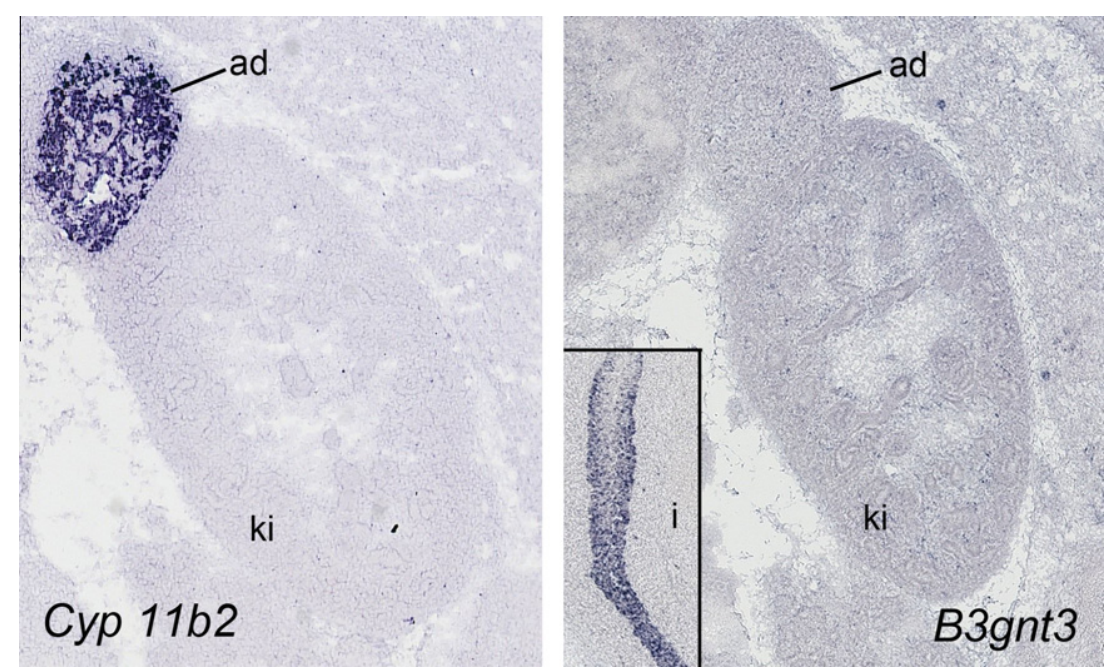

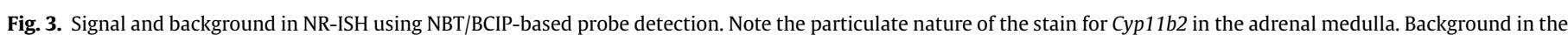

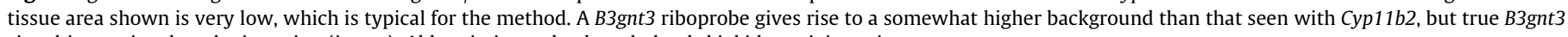
signal is restricted to the intestine (insert). Abbreviation: ad, adrenal gland; ki, kidney; i, intestine. 
"b") located on the robot platform. Even solutions used at room temperature must always be equilibrated rather than be taken out of the refrigerator and immediately put onto the platform for use. This also applies to the tyramide amplification solution which needs to be freshly made on day 2 but must be prewarmed to RT for half an hour. Solutions containing antibody conjugates should be gently mixed to avoid bubble formation.

\subsection{Additional issues}

When efficiently used, the high-throughput ISH protocol described above generates large amounts of expression patterns. These need to be scanned in an automatically using a microscope equipped with an automated slide loader and an automated scanning stage. Only a few years ago, this required the development of customized equipment (e.g. [9]), but such equipment is now commercially available, even for fluorescence-based detection. Since large quantities of image data will be produced (easily in the $50 \mathrm{~TB}$ range) there is a need for proper and secure archiving and for conversion of the primary images (TIFF or bitmaps) to a format that can be viewed through the internet using zoom-in features.

The generation of a large number of gene expression pattern images also requires a thorough data management. This is optimally achieved with a database (Laboratory Information Management System, LIMS) but, in case of a more limited amount of data, Excel spreadsheets may be adequate for data tracking. The purpose of a database is to collect metadata associated with a particular gene expression pattern and to establish a data stream that avoids any mix-up of experimental data. Metadata include sample identity, riboprobe identity, probe sequence, link to particular protocols used, laboratory staff names, date of the various procedures carried out etc. Moreover, the massive amount of image and metadata accrued in such experiments should be readily retrievable, either from an in-house database or a web database. Project-specific information and data can then be linked to other databases that provide information on gene sequence and structure, literature related to the genes investigated, etc. Eurexpress.org, for example, provides user interfaces for viewing expression patterns through the internet and also contains numerous links to other public web sites for each and every gene analyzed [10].

Discriminating between signal and background can be a complication with weakly expressed genes. It is paramount to include in each ISH run a positive control that yields strong staining as well as a blank. Generally, tissue-characteristic marker genes are suitable positive controls. For example, for brain sections and for sections of embryos containing nervous system tissues, a neuropeptide $Y$ riboprobe is a good positive control since this gene is strongly expressed in a distinct pattern throughout the nervous system. For skin, one can use Indian hedgehog and for liver albumin etc. A suitable negative control is the omission of riboprobe. In our experience, the absence of signal for an authentic gene marker frequently indicates a problem in one of the reagents used. Seeing a signal in the negative control indicates high background staining. A characteristic feature of the NBT/BCIP color reaction is the formation of a particulate precipitate that can readily be seen in a microscope at higher magnification. By contrast, background staining is (mostly) diffuse and devoid of grains. The grainy nature of the dye is readily seen for the adrenal staining of Cyp11b2 (Fig. 3). Although the adrenal can be recognized in sections hybridized with the B3gnt3 riboprobe (Fig. 3), the signal is diffuse rather than grainy. A B3gnt3 signal proper is seen in the intestine (insert in Fig. 3).

In conclusion, commercially available instrumentation and robust protocols for semi-automated NR-ISH have been developed and reported by the web databases that store such data such as Eurexpress, the Allen Brain Atlas and Genepaint. The automation of NR-ISH can be readably extended to dual and triple labeling approaches using fluorescent dye indicators. In this case, the process involves many more washing and incubation steps, thus benefiting from a high degree of automation and process control. The use of the methods describe here is by no means restricted to the construction of gene expression atlases. In situ hybridization is a valuable tool for validation of findings from high-throughput screens such as RNA interference [11] and prediction of signaling cascades that require coexpression of genes in the same tissue [12].

\section{Acknowledgments}

We thank Frauke Grabbe, Katharina Jaeckle, Christina Thaller, Murat Yaylaoglu and Sandro Banfi for their assistance in the preparation of this manuscript. This work was supported by the EC VI Framework Programme contract number LSHG-CT-2004-512003. We acknowledge the support of: the Max Planck Society (G.E.) and the Italian Telethon Foundation (G.D.-R.).

\section{References}

[1] M.L. Pardue, Annu. Rev. Cell. Dev. Biol. 23 (2007) 1-22.

[2] J.P. Carson, G. Eichele, W. Chiu, J. Microsc. 217 (2005) 275-281.

[3] C.M. Cremer, M. Cremer, J.L. Escobar, E.J. Speckmann, K. Zilles, J. Neurosci. Methods 185 (2009) 56-61.

[4] E.S. Lein, M.J. Hawrylycz, N. Ao, M. Ayres, A. Bensinger, A. Bernard, A.F. Boe, M.S. Boguski, K.S. Brockway, E.J. Byrnes, L. Chen, T.M. Chen, M.C. Chin, J. Chong, B.E. Crook, A. Czaplinska, C.N. Dang, S. Datta, N.R. Dee, A.L. Desaki, T. Desta, E. Diep, T.A. Dolbeare, M.J. Donelan, H.W. Dong, J.G. Dougherty, B.J. Duncan, A.J. Ebbert, G. Eichele, L.K. Estin, C. Faber, B.A. Facer, R. Fields, S.R. Fischer, T.P. Fliss, C. Frensley, S.N. Gates, K.J. Glattfelder, K.R. Halverson, M.R. Hart, J.G. Hohmann, M.P. Howell, D.P. Jeung, R.A. Johnson, P.T. Karr, R. Kawal, J.M. Kidney, R.H. Knapik, C.L. Kuan, J.H. Lake, A.R. Laramee, K.D. Larsen, C. Lau, T.A. Lemon, A.J. Liang, Y. Liu, L.T. Luong, J. Michaels, J.J. Morgan, R.J. Morgan, M.T. Mortrud, N.F. Mosqueda, L.L. Ng, R. Ng, G.J. Orta, C.C. Overly, T.H. Pak, S.E. Parry, S.D. Pathak, O.C. Pearson, R.B. Puchalski, Z.L Riley, H.R. Rockett, S.A. Rowland, J.J. Royall, M.J. Ruiz, N.R. Sarno, K. Schaffnit, N.V. Shapovalova, T. Sivisay, C.R. Slaughterbeck, S.C. Smith, K.A. Smith, B.I. Smith, A.J. Sodt, N.N. Stewart, K.R. Stumpf, S.M. Sunkin, M. Sutram, A. Tam, C.D. Teemer, C. Thaller, C.L. Thompson, L.R. Varnam, A. Visel, R.M. Whitlock, P.E. Wohnoutka, C.K. Wolkey, V.Y. Wong, M. Wood, M.B. Yaylaoglu, R.C. Young, B.L. Youngstrom, X.F. Yuan, B. Zhang, T.A. Zwingman, A.R. Jones, Nature 445 (2007) 168-176.

[5] S. Magdaleno, P. Jensen, C.L. Brumwell, A. Seal, K. Lehman, A. Asbury, T. Cheung, T. Cornelius, D.M. Batten, C. Eden, S.M. Norland, D.S. Rice, N. Dosooye, S. Shakya, P. Mehta, T. Curran, PLoS Biol. 4 (2006) e86.

[6] E.J. Speel, Histochem. Cell. Biol. 112 (1999) 89-113.

[7] E.J. Speel, P. Komminoth, Endocr. Pathol. 10 (1999) 193-198.

[8] M.B. Yaylaoglu, A. Titmus, A. Visel, G. Alvarez-Bolado, C. Thaller, G. Eichele, Dev. Dyn. 234 (2005) 371-386.

[9] J.P. Carson, C. Thaller, G. Eichele, Curr. Opin. Neurobiol. 12 (2002) 562-565.

[10] Diez-Roux G, Banfi S, Sultan M, Geffers L, Anand S, et al. 2011 A HighResolution Anatomical Atlas of the Transcriptome in the Mouse Embryo. PLoS Biol 9(1): e1000582. doi:10.1371/journal.pbio.1000582.

[11] S. Mohr, C. Bakal, N. Perrimon, Annu. Rev. Biochem. 79 (2010) 37-64.

[12] C. Niehrs, N. Pollet, Nature 402 (1999) 483-487. 